\title{
The relationship between mental computation and relational thinking in the seventh grade
}

\author{
Alexandra N. Kindrat ${ }^{*}$ and Helena P. Osana
}

*Correspondence:
a_kindra@education.
concordia.ca
Department of Education,
Concordia University, 1455
de Maisonneuve Blvd. Ouest,
Montréal, QC H3G 1M8,
Canada

*Correspondence:

a_kindra@education.

Department of Education,

Concordia University, 1455

Montréal, QC H3G 1M8,

Canada

\begin{abstract}
Relational thinking involves understanding equivalence and numerical relationships. The present study examined the relational thinking of seventh graders before and after a 15-day mental mathematics intervention in the context of whole number arithmetic. Using two intact seventh-grade classes and a staggered treatment design, students were assessed at three time points on their (a) ability to solve equivalence problems, and (b) reasoning abilities about true-false number sentences. The results indicated that the students in the class that received the intervention first (the Intervention First group) improved their performance on both measures after the intervention, and a similar pattern was found for the second class (the Intervention Second group), indicating that each group improved immediately following the mental mathematics intervention. Students in the Intervention First group were able to maintain their scores on the test of equivalence problems 4 weeks after the conclusion of the intervention. As the results suggest a link between mental mathematics and relational thinking, it is recommended that mental mathematics play a more prominent role in the seventhgrade mathematics classroom.
\end{abstract}

Keywords: Relational thinking, Equal sign, Mental mathematics, Algebraic reasoning, Mathematics instruction

\section{Introduction}

Among the many concepts included in the middle- and secondary-school curricula is algebra, a topic that often causes much difficulty for many students [8, 17, 33]. As algebra is known to be a gatekeeper to students' future academic and professional opportunities $[22,41]$, alleviating their struggles in algebra is of critical importance. While algebra allows for mathematical generalizations and solving for unknown quantities, critical to its success is its precursor, algebraic reasoning. Algebraic reasoning has been conceptualized as attending to patterns and rules in creating mathematical generalizations [8], and is important because it allows for an understanding of the structure of mathematics. Individuals who are successful in algebraic reasoning are able to discover patterns in a variety of mathematical expressions, and further generalize these patterns from familiar to unfamiliar situations. 
In an attempt to aid students in their algebraic success, mathematics educators have argued that exposure to algebraic reasoning should begin in kindergarten and thread through the mathematics curriculum to the end of high school [11,33]. Algebraic reasoning is accessible to children as early as kindergarten and finds a natural home in the early primary grades, when students are first introduced to numbers, basic arithmetic, and the equal sign [31,33], allowing for the integration of arithmetic and algebra. For example, the mathematical relationships in arithmetic expressions such as $8+\square=12$ could be used to reflect on $1986+8+\square=1986+12$, and eventually to $x+8+y=x+12$. Encouraging students to reflect on the numerical relationships between structurally similar expressions promises a seamless transition from arithmetic to algebra. Success in algebra, therefore, is predicated on instruction of arithmetic in ways that supports students' algebraic reasoning skills and creates a solid foundation for learning algebra with meaning [8].

At the center of algebraic reasoning is relational thinking, which involves coordinating quantities in a mathematical expression, often without computation, using flexible reasoning about quantities and transforming mathematical expressions into equivalent ones. Transformations of numerical expressions are governed by implicit or explicit understandings of mathematical properties and relational views of the equal sign [10, 44]. Referring to the previous example of $1986+8+\square=1986+12$, for instance, students with a relational view of the equal sign are able to determine that the answer is 4 by reflecting on the relationship between the numbers - the equal sign permits students to disregard the 1986 on each side and focus only on the resultant equation $8+\square=12$, which would either reduce the level of computation or allow it to be circumvented altogether. Because success in algebraic reasoning can be seen as linked to one's abilities in relational thinking [31, 43], instruction to improve algebraic reasoning should focus on relational thinking. Despite the apparent relationship between algebraic reasoning and relational thinking, the literature on teaching for algebraic reasoning through relational thinking is incomplete. In an attempt to understand how relational thinking instruction might be improved, we noticed in much of the mathematics education literature that both relational thinking and mental mathematics share several features $[17,44,46,47]$. As such, the aim of this study was to determine whether relational thinking could be improved by means of a mental mathematics intervention as a vehicle for improving relational thinking in the seventh grade.

\section{Relational thinking}

We adopted the theoretical framework forwarded by Jacobs et al. [17], who defined relational thinking as a form of reasoning that entails looking at numbers and expressions holistically and noticing relations among them. The central feature of relational thinking hinges on understanding the meaning of the equal sign ("="; $[12,22]$ ), which indicates a relationship of equality between numbers or expressions [ $[8,17]$. To engage successfully in relational thinking, students must first consider the number sentence as a whole, and then uncover relevant components and numerical relationships [32]. Without accurate conceptions of the equal sign in both canonical (e.g., $3+4=7$ ) and noncanonical (e.g., $3+4=2+5$ ) contexts, there is nothing upon which relational thinking can be based. Because misconceptions about the equal sign have been well documented $[4,5,11,19$, 
$26,27,38]$, relational thinking is often compromised, even at the middle-school level, where the operator view remains prevalent $[19,21,42]$.

To illustrate, when faced with a problem such as $25+17=22+\square$, students who understand the equal sign to mean "the same as" could solve it via computation (i.e., $25+17-22$ ) or by reasoning relationally, which would entail examining the relationship between the amounts on both sides of the equation (i.e., " 25 is 3 more than 22 , so the answer must be 20 because to balance it out I would need a number 3 more than 17."). In this context, then, relational thinking involves examining the equation, noticing the structural connection between the 25 on the left side of the equation and the 22 on the right side, and compensating for the discrepancy by adjusting the 17 accordingly. The hallmark of relational thinking is not determined by computation, but rather on mathematical structures and generalizations [9, 44].

Furthermore, students who think relationally engage in transformations that are justified, often implicitly, by properties of whole number operations. When asked to think relationally about $99 \times 3$, for example, students can, and often do (e.g., [2]), use the distributive property by transforming 99 into $(100-1)$, so the product can then be computed by subtracting 3 from 300 (i.e., $99 \times 3=(100-1) \times 3=300-3=297)$. Such transformations rely on the notion of substituting 99 for $(100-1)$, a key element to understanding the equal sign [18]. Thus, each transformation results in an expression that is mathematically equivalent to the first (in this case, $(100-1) \times 3$ and $300-3$ are both equivalent to $99 \times 3$ ). In sum, fostering relational thinking is critical because it gives meaning to arithmetic, leads to conceptual understanding of number and number properties, and enhances students' thinking about mathematical generalizations [17, 32, 43].

Difficulties with the equal sign and relational thinking more broadly are not confined to the early grades. At the sixth-grade level, it has been shown that a majority of students have trouble finding the missing number that would make a noncanonical expression, such as $8+4=\square+5$, true $[11,19,31]$. In fact, Falkner et al. [11] found that of the 145 participating sixth graders, none correctly placed the number 7 in the box in the above expression (similar results were revealed by Li et al. [23]). Further, Knuth et al. [22] asked students from the sixth to the eighth grade to name and define the equal sign in the expression $3+4=7$. The authors found that only $32 \%$ of the sixth graders, $43 \%$ of the seventh graders, and $31 \%$ of the eighth graders offered a relational definition of the equal sign. Such difficulties with the equal sign may be due to the almost exclusive use of canonical equations in student textbooks $[7,28]$. In an evaluation of four middle-school textbooks, McNeil et al. [28] concluded that the infrequent use of noncanonical equations may be responsible for reinforcing students' operator view of the equal sign at the middle-school level [28].

Possibly because of their misconceptions about the equal sign, students at the middleschool level also struggle with relational thinking more generally. Stephens and Ribeiro [44] asked the seventh- and the eighth-grade Brazilian students to find numbers to make equations with two unknowns true (e.g., $18+\square=20+\square$ ). Because such open-number sentences have more than one correct answer, they emphasize the relationship between the quantities on both sides of the equal sign and as such, specifically target structural thinking rather than computation. The authors also asked the students follow-up questions such as, "When you make a correct sentence, what is the relationship between the 
numbers in [the two boxes]?", p. 385; and "If instead of 18 and 20, the first number was 226 and the second number was 231, what would be the relationship between numbers in [the two boxes]?", p. 385. The authors used a descriptive scale to score the students' responses to the follow-up questions that ranged from fully nonrelational to fully relational. The data revealed that the majority of students were not able to offer a fully relational response to the items and several students provided incorrect descriptions of the quantitative relationship between the numbers in the boxes (e.g., "The second box will always be bigger than the first box."). A similar lack of relational thinking was observed in the sixth graders from both Australia and China [45].

\section{Mental mathematics}

Mental mathematics, sometimes referred to as mental computation, is the act of performing calculations without the use of external tools through a sequence of transformations beyond counting or imagining the steps of the standard algorithm [24, 37, 46]. Robust cognitive models of mental computation are less prevalent than models of relational thinking, but several theoretical frameworks involve two major processes: (a) the selection of a strategy that would make the computation more manageable, and (b) the execution of the calculations once the strategy is chosen [24, 40, 47].

A child faced with a mental computation problem must first decide how to transform the numbers in ways that will reduce cognitive load when computing. The transformation is often achieved through implicit or explicit knowledge of numbers and number properties [2, 47]. For example, faced with $113-30$, a child could decide to transform 30 into $10+10+10$ because removing 10 three times is more manageable than removing 30 at once. Maclellan [24] argued that mental computation entails transforming expressions into others that look different but are not changed in value (our emphasis). In this way, a child performing $113-30$ is essentially relying simultaneously on both notions of sameness and substitution: $113-30$ is the same as and can be substituted for $113-(10+10+10)$, which is the same as and can be substituted for $113-10-10-10$. We argue, therefore, that choosing a mental computation strategy is an act of relational thinking because it relies on creating implicit transformations that rely squarely on both "sameness" and substitution principles of mathematical equivalence [18]. Once a child decides what strategy to use, he or she must then mentally perform the requisite arithmetical operations that follow from the selected strategy, which also rely on number knowledge, such as basic facts and place value [24, 40]. In the context of the current example, these operations would amount to: $113-10=103 \rightarrow 103-10=93 \rightarrow 93-10$ $=83$.

In sum, existing models of mental computation incorporate processes that are strikingly similar to those described in the relational thinking literature. Unlike mental computation, however, conceptualizations of relational thinking are not centered on computation or calculation, primarily because of the theoretical overlap with algebraic reasoning, which is based on structural reasoning about mathematical generalizations. Therefore, we see relational thinking as implicated in the selection and execution of mental computation strategies, and as such, is a central component of mental computation. Relational thinking is not the only process involved in mental computation, however. The goal of a mental computation problem is to arrive at an answer, and consequently, 
mental calculations are required. Performing mental calculations may take place during relational thinking, but it is not one of its defining elements.

\section{Improving relational thinking through instruction}

Because of apparent overlap in cognitive processes required for relational thinking and mental computation, one might hypothesize that instruction that targets fluency in mental computation would enhance children's conceptions of the equal sign and their relational thinking more generally. A handful of studies provide evidence to support this hypothesis. Irwin and Britt [16], for instance, described the Numeracy Project as an effective curriculum for improving relational thinking in New Zealand. The Numeracy Project is a mathematics curriculum designed for primary-school students (ages 5-14) to improve their understanding of operations and to assist them to be flexible in their problem-solving strategies. Students participating in the Numeracy Project were encouraged to use a variety of mental strategies to solve mathematics problems such as $27+15$ or $34+19$, rather than rely on computation or paper-and-pencil algorithms. For example, students could transform $27+15$ into $30+12$ to make the mental computation less arduous [6]. Strategies not limited to "compensating, factorising, and maintaining equivalence" ([16], p. 170) were reinforced during the program, and students were exposed to applying the above and similar strategies before becoming acquainted with any algebraic symbols.

When comparing assessments of 12-year-old students who participated in the Numeracy Project for at least one year to those who did not, students who participated in the Numeracy Project used techniques associated with relational thinking more often on an assessment that evaluated compensation in addition, subtraction, and multiplication, as well as equivalence between whole number and fractional quantities [16]. These results suggest, in line with our hypothesis, that improvements in mental mathematics may be related to students' relational thinking. Because Irwin and Britt's methodology did not allow them to explain the students' improvement other than that they participated in the Numeracy Project, further research is required to investigate the mechanisms that may have been responsible for the increases in relational thinking.

In possibly the most relevant study for our purposes, Osana et al [34] carried out a mental mathematics unit in a university-level elementary mathematics methods course for preservice teachers that consisted of mental mathematics activities, discussion, and practice problems. The authors administered a written test of relational thinking before and after the 3-week mental mathematics unit. The authors' hypothesis was that the students' relational thinking scores would improve after having engaged in the mental mathematics activities. Indeed, their predictions were borne out: The students' relational thinking improved, but again, methodological weaknesses in their study, such as the absence of a control group, prevented them from explaining their results. In addition, the authors' study only involved university level students, and as such, the generalizability of their findings to seventh graders is tentative at best. 


\section{Present study}

The present study investigated the impact of a mental mathematics intervention on the seventh graders' relational thinking and their understanding of the equal sign. The seventh graders were selected as participants for the present study because of the relative lack of research on relational thinking instruction at the middle-school level, the point at which formal algebra instruction begins. Using two intact seventh-grade classes, students were assessed at three time points (Time 1, Time 2, and Time 3) on their (a) understanding of the equal sign symbol, as operationalized by the ability to solve equivalence problems, and (b) their relational thinking, operationalized by the ability to reason structurally about true-false number sentences.

When students are trained in mental mathematics, they practice relational thinking by performing substitutions of equivalent expressions in their head. Because such transformations are based on sameness and substitution, we hypothesized that their interpretations of the equal sign would improve. The theoretical underpinnings of mental mathematics would also allow us to hypothesize that practice in mental transformations would lead to a stronger conceptual grasp of number properties that would justify said transformations (see $[25,40]$ ). This would consequently support students' judgments of the noncanonical expressions in subsequent relational thinking tasks. We therefore predicted that following the mental mathematics intervention in each class, students' understanding of the equal sign and their relational thinking would improve. We also predicted that the performance of the students in the first class would be maintained from Time 2 to Time 3.

\section{Methods}

\section{Participants}

The seventh-grade students from two intact classes from a suburban public English speaking high school in Quebec, Canada ${ }^{1}$ were initially asked to participate in the study. Twenty-four students from the first class and 28 students from the second class were invited. The final sample consisted of 20 students from the first class (hereafter referred to as the Intervention First group) and 24 students from the second class (the Intervention Second group). Of the eight students who were excluded, one was transferred to another class, two did not return the consent form, one did not have parental consent, and the remaining four students did not complete all of the assessments.

We did not have access to the exact ages of the students, but all participants were at the government-mandated age for the seventh graders, that is, from 11 years and 11 months to 12 years and 11 months. All students were part of the French Immersion program at the high school, but all mathematics instruction was in English. In the Intervention First group, there were 12 males and 8 females, and in the Intervention Second group there were 15 males and 9 females.

The high school was composed of middle- to high-income families, and was rated on the higher end of the school board's socio-economic index, measured by family income

\footnotetext{
${ }^{1}$ In Quebec, elementary students move to high school after the sixth grade. High school begins in the seventh grade, and students graduate after the eleventh grade. Thus, although the participants were at the middle-school level in terms of age, in practice, they were attending a high school.
} 


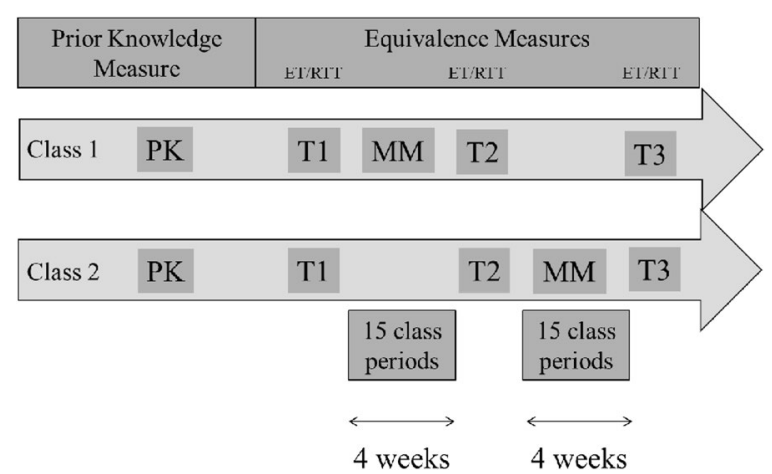

Fig. 1 Design of the Study. T1 Time 1, T2 Time 2, T3 Time 3, PK Prior Knowledge test, ET Equivalence Test, RTT Relational Thinking Test, $M M$ mental mathematics intervention

levels and mother's education [29]. Both classes followed the identical seventh-grade mathematics curriculum, as mandated by the Quebec Education Plan of the Ministère de l'éducation, enseignement supérieur, et recherche [30]. Canadian Mathematics 7 [35] was used as the textbook for the delivery of the curriculum.

\section{Design}

We used a multiple baseline design, presented in Fig. 1. A paper-and-pencil test of prior mathematics knowledge (PK) was first administered to ensure that no differences existed between the two classes. The next day, at Time 1, two assessments, the equivalence test (ET) and relational thinking test (RTT), were administered, in that order, to both classes prior to any intervention. The following day, the mental mathematics (MM) intervention began in the Intervention First group. The MM instructional sessions took place in the first 20 min of each of 15 mathematics classes over a 4-week period. During these 4 weeks, the students in the Intervention Second group did not receive any MM instructional sessions, but rather received the regular seventh-grade curriculum. The day after the MM intervention was completed in the Intervention First group, isomorphic versions on the ET and RTT assessments were administered at Time 2 to both classes. The following day, the MM intervention began in the Intervention Second group using the same procedures, while the Intervention First group received the regular curriculum. The day after the second delivery of the intervention, isomorphic versions of the ET and RTT assessments were then administered a third time, at Time 3, to all students in both classes.

\section{Measures}

We administered three assessments: the prior knowledge test (PK), the equivalence test (ET), and the relational thinking test (RTT). The PK test was designed to assess the students' procedural knowledge in arithmetic and their skills working with exponents, converting decimals to fractions, and ordering rational numbers. The ET was designed to measure the students' understanding of the equal sign (based on Sherman and Bisanz [39] and used by Falkner et al. [11]; and Perry [36], with older students), and the RTT was designed to assess students' ability to determine the truth value of equations using relational thinking. Students who were absent completed their assessments immediately 
upon their return, up to three days after the official assessment day, provided their class intervention had not yet begun.

At the start of each day of testing (day 1: PK; day 2: ET and RTT), the teacher indicated to the students that they would be completing tests. She told them that the assessment(s) would not count in terms of their mathematics course grade, but that they should complete the tests to the best of their ability and to take the exercise seriously. No feedback or clarification was provided to any student at any time during the assessments, and the students completed the tests independently.

\section{Prior knowledge test (PK)}

The PK was a paper-and-pencil multiple-choice measure consisting of 16 items, and was used to assess the students' knowledge of the sixth-grade mathematics curriculum. The assessment consisted of procedural knowledge items because they were similar to the types of mental computation skills required of the students during the intervention. Specifically, the assessment comprised two whole number computations requiring knowledge of the order of operations (e.g., $14+3 \cdot 8$ ), six decimal number (e.g., $.46+.72=$ ) and two fractions (e.g., $5 / 8+5 / 12=\ldots$ ) computation questions, two exponent questions (e.g., $24=\ldots$ ), two questions requiring conversion from decimals to fractions (e.g., Which of the following decimals is equivalent to 6/25?), and two questions asking students to select the greatest number from a set of four rational numbers. All computation questions were presented horizontally, and the students were required to circle their response from a list of four choices.

At the start of the PK test, the teacher delivered a paper copy to each student, placing it face down on his or her desk. Once every student had a test, the students were asked to turn them over, and the teacher stated that the class had $30 \mathrm{~min}$ to complete the PK. The students were permitted to use the margins for computations and other written work, but the teacher told them that only their multiple-choice selections would be graded. The teacher also instructed the students to look over their answers once they were finished, and to remain silent for the duration of the assessment. Calculators were not permitted. After the 30 min were completed, students were asked to turn over their papers, and the teacher collected them.

Correct answers received 1 point and incorrect answers 0 points. The points were summed to obtain a total PK score, which was out of a possible 16 points. The scores were then converted to percent. Cronbach's alpha reliability estimate for the PK was .67.

\section{Equivalence test (ET)}

The ET is a paper-and-pencil test created by Sherman and Bisanz [39] designed to assess students' interpretation of the equal sign symbol. The test consisted of 29 problems, including 9 canonical and 20 noncanonical problems, and each contained only single-digit numbers. The canonical items involved addition and subtraction and the noncanonical problems involved only addition. Examples of noncanonical problems were: $7+8=6+\ldots, 7+3=7+\ldots, 4+7=7+_{\ldots}$. A different isomorphic version of the ET was used at each time point. Each version contained the same number of each type of problem (i.e., four identity, $a+b=a+_{-}$or $a+b={ }_{-}+b$; four commutativity, $a+b=b+\ldots$ or $a+b=\ldots+a$; eight part-whole, $a+b=c+\ldots$ or $a+b+c=\ldots+d$; and 
four combination, $a+b+c=a+\ldots$ or $a+b+c=\ldots+a$; see Sherman and Bisanz [39], for a description of these problem types). The numbers in the problems and the order of the problem types varied in each version.

At the start of testing, the teacher delivered the ET to each student, placing it face down on his or her desk. Once every student had a test, the teacher went through the instructions orally in front of the class. Specifically, she stated that the class had $15 \mathrm{~min}$ to complete the ET, and that they were to complete the assessment by writing down their answer on a blank line provided in each equation. The teacher also instructed the students to look over their answers once they were finished, and to remain silent for the duration of the assessment. After the 15 min were completed, students were asked to turn over their papers, and the teacher collected them.

Only the responses for the 20 noncanonical problems were used in the analyses. Correct answers received 1 point and incorrect answers 0 points. The points were summed to obtain a total ET score, which was out of a possible 20 points. The scores were then converted to percent. Cronbach's alpha reliability estimates for the ET was .99 at Time 1, .98 at Time 2, and .98 at Time 3.

\section{Relational thinking test (RTT)}

The RTT is a paper-and-pencil test based on Osana et al. [34] and Carpenter et al. [8] and designed to assess the degree to which students use relational thinking, as opposed to computation, to judge the truth value of noncanonical equations. There were four items on the RTT, one for each operation (addition, subtraction, multiplication, and division) and each item consisted of a number sentence, such as $228 \div 6=456 \div 12$. The students were asked to indicate whether the sentence was true or false by circling the word "true" or "false" on the test paper. The students were then asked to provide a written justification for their responses in a blank space provided on the test. Examples of RTT items are: $65+36=67+38,105-45=106-46,228 \div 6=456 \div 12$, and $29 \times 52=28 \times 53$.

Three isomorphic versions of this assessment were administered for the repeated administrations across the study. In each version, all four operations were used, but the order of the items varied across versions. The numbers used in each version were also different, but the structure of the numerical relationships across versions remained the same for each operation (e.g., Version 1: $67+48=65+46$; Version 2: $55+36=53+34$; Version $3: 73+57=71+55)$.

Immediately following the collection of the ET, the teacher delivered the RTT to each student. The students were given 20 min to complete the RTT because they were required to provide written justifications for their answers. Again, the teacher reviewed the instructions orally with the class. She instructed the students to indicate in each question if the number sentence was true or false and to justify their answers in the space provided. The students were also told that they were not permitted to communicate during the test. After $20 \mathrm{~min}$, students were asked to turn over their tests, and the teacher collected them.

Only the students' written justifications were coded using the following rubric: (a) Category 1: Relational thinking without computation or with computation only as a means to justify a written relational response; (b) Category 2: Computational; (c) Category 


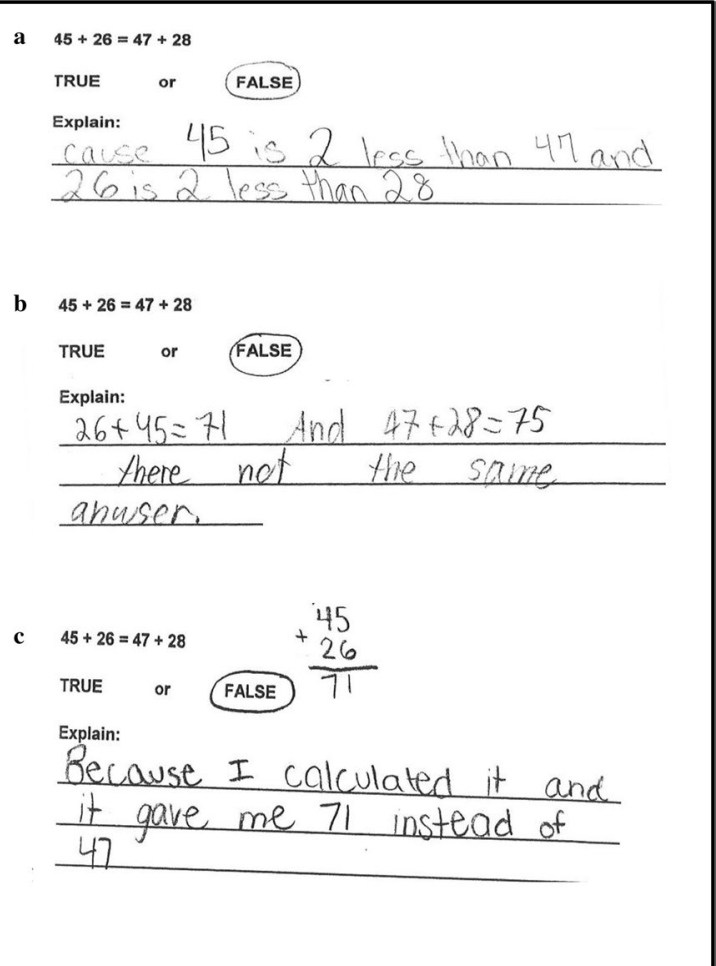

Fig. 2 Relational thinking coding examples. a Category 1: relational thinking without computation or with computation only as a means to justify a written relational response; b Category 2: computational; c Category 3: other

3: Other. Examples of students' responses for each category can be found in Fig. 2. Responses that were placed in Category 1 demonstrated that the student engaged in relational thinking by considering the relationship between the numbers without computing the quantities on both sides of the equal sign to determine the truth value of the equation. Computation in this category was permitted only if the student had first justified the response relationally and only if the computation was used to support or illustrate the relational response. Students' responses that were placed in Category 2 demonstrated that the student had an understanding of the equal sign symbol and that they were able to determine if the response was true or false with the use of computation only. Category 3 responses were those where the student either had an operator view of the equal sign, did not supply any justification, or provided responses that were impossible to interpret.

Category 1 responses received 2 points, Category 2 responses received 1 point, and Category 3 responses received 0 points. Category 1 responses were awarded more points than Category 2 and 3 responses because they indicated that the students responded relationally and did not need to compute to arrive at their answer. Category 2 responses were considered relational as opposed to operational, because the students appeared to understand the meaning of the equal sign, but used computation to justify their responses. Category 3 responses received 0 points because they contained no evidence of relational thinking or an understanding of the equal sign. 
Item scores were summed for a total RTT score that ranged from 0 to 8 . Total scores were then converted to percents. A random sample of $20 \%$ of the responses was coded by a second rater, and inter-rater reliability of $90 \%$ agreement was achieved. Cronbach's alpha reliability estimates for the RTT was .83 at Time $1, .84$ at Time 2 , and .80 at Time 3 .

\section{Mental mathematics intervention}

\section{Intervention tasks}

Before the study began, we created 26 sets of expressions for the mental mathematics intervention. Each set contained four expressions, one for each operation. The first set, for example, contained the expressions $62+38 ; 73-31 ; 21 \times 9$; and $225 \div 25$. The second set of expressions contained the same four operations, but presented in a different order, such as $77-26 ; 17 \times 5 ; 600 \div 4$; and $42+58$. Each subsequent set contained all four operations in a different order from the preceding set. This ensured that the discussion time for each operation over the 15-day intervention was as similar as possible within and across classes. The teacher began the intervention in each class by starting with Set 1 and continued across the 15 sessions through as many of the 26 sets as possible. In each mental mathematics session, she continued through the sets until 20 min were completed. During the next session, she picked up where she had left off in the previous session and again continued through the sets in the specified order. In any given session, between six and seven expressions were computed mentally and discussed. Across the 15-day intervention, 92 mental mathematics expressions were used for the Intervention First group, which included 23 addition problems, 22 subtraction problems, 23 multiplication problems, and 24 division problems. For the Intervention Second group, 94 mental mathematics expressions were used, which included 24 addition problems, 23, subtraction problems, 24 division problems, and 23 multiplication problems.

\section{Instructional procedure}

The intervention for both classes was delivered by the first author, who was the students' regular mathematics teacher. At the beginning of each MM instructional session, the students were seated at their own desks with their own individual white board and dry erase marker. No other materials were provided. The teacher began the session by writing a mathematical expression on the white board (e.g., $37+58)$ and then gave the students $30 \mathrm{~s}$ to compute the answer mentally. The students were instructed to remain silent during the $30 \mathrm{~s}$ and not to write anything down but their final answer on their white boards. Once the 30-s time period was up, the teacher asked the students to hold up their white boards so that she could view the answers.

After raising their white boards, the students then shared their strategies with their peers during whole-class discussions, with guidance from the teacher. First, the teacher asked a student with an incorrect answer to describe how he or she had reached the answer. The teacher then asked a student who had computed a correct answer to describe his or her strategy. The discussion centered on both incorrect and correct responses, with students discussing the merits of one strategy relative to another. The discussion for each expression lasted no more than $4 \mathrm{~min}$, during which time the equal sign was never displayed. The teacher then erased the mathematical expression from the board, and the session continued with the next mathematical expression. 
During the discussions, the teacher pointed out how the students rearranged, transformed, and substituted numbers to make the computations easier to compute mentally. In addition, she pointed out that certain strategies were particularly suitable for specific operations. For example, she illustrated how dividing large numbers by factors of the divisor made it easier to divide mentally, and she also encouraged students to estimate what their answer should be before they used any strategy. In addition, the teacher highlighted applications of the fundamental properties of arithmetic without directly naming them. For example, while discussing $22 \times 6$, the teacher underscored how the distributive property was used in one of the student's strategies. Specifically, she explained how the 6 was substituted for $4+2$, yielding $22 \times(4+2)$, which then resulted in the sum of two products, $22 \times 4$ and $22 \times 2$, yielding $88+44$. The latter computation was also discussed; the student had transformed it into the equivalent expression of $80+40+8+4$, ultimately leading to 132 as a final answer.

\section{Results}

For each measure (ET and RTT), we computed correlations between administrations at different times points to determine test-retest reliability. The correlations were computed separately for each class because the intervention was delivered at different times for each group. Suitable comparisons were those not affected by the intervention, namely between Time 2 and Time 3 for the Intervention First group and between Time 1 and Time 2 for the Intervention Second group. For the Intervention First group, the 4-week test-retest reliability coefficient for the ET was .87 and for the RTT was .66. For the Intervention Second group, the 4-week test-retest reliability coefficient for the ET was .78 and for the RTT it was.13. Thus, there is support for test-retest reliability for the ET for both classes, but not for the RTT for the Intervention Second group.

\section{Performance as a function of the MM intervention}

The means and standard deviations of the prior knowledge test (PK), the equivalence test (ET), and the relational thinking test (RTT) scores as a function of class and time are presented in Table 1. A $t$ test revealed no difference in prior knowledge between the Intervention First group and the Intervention Second group at Time $1, t(42)=.68$, $p=.50$. Thus, there were no differences in prior mathematics knowledge between the two classes, at least with respect to what was assessed on the PK.

\section{Equivalence test}

We ran a 3-Time (Time 1, Time 2, Time 3) $\times 2$-Intervention Group (Intervention First, Intervention Second) $\times 4$-Problem Type (identity, commutativity, part-whole, and combination) mixed ANOVA, with time and problem type as the within-groups factors. No effects of problem type were found except for a main effect, $F(3126)=5.07, p=.002$, partial $\eta^{2}=.11$, which is uninterpretable in this context given the means are averaged across both groups and all three time points. A graphical representation of the ET means as a function of time and group, collapsed across problem type, is presented in Fig. 3.

We conducted a 3-Time (Time 1, Time 2, Time 3) $\times 2$-Intervention Group (Intervention First, Intervention Second) using the mean ET scores collapsed across problem type as the dependent measure. Intervention group was the between-groups factor, 
Table 1 Means and (standard deviations) of Prior Knowledge test (PK), Equivalence Test (ET), and Relational Thinking Test (RTT) as a function of intervention group and time

\begin{tabular}{|c|c|c|c|c|c|c|c|}
\hline & \multicolumn{3}{|l|}{ T1 } & \multicolumn{2}{|l|}{ T2 } & \multicolumn{2}{|l|}{ T3 } \\
\hline & PK & ET & RTT & ET & RTT & ET & RTT \\
\hline Intervention First group ${ }^{\mathrm{a}}$ & $.42(.23)$ & $.54(.44)$ & $.51(.20)$ & $.96(.10)$ & $.79(.22)$ & $.93(.22)$ & $.69(.22)$ \\
\hline Intervention Second group ${ }^{\mathrm{b}}$ & $.38(.16)$ & $.57(.45)$ & $.45(.24)$ & $.73(.40)$ & $.49(.19)$ & $.94(.20)$ & $.58(.21)$ \\
\hline
\end{tabular}

All measures scores are reported in percents

T1 Time 1, T2 Time 2, T3 Time 3

a $N=20$

b $N=24$

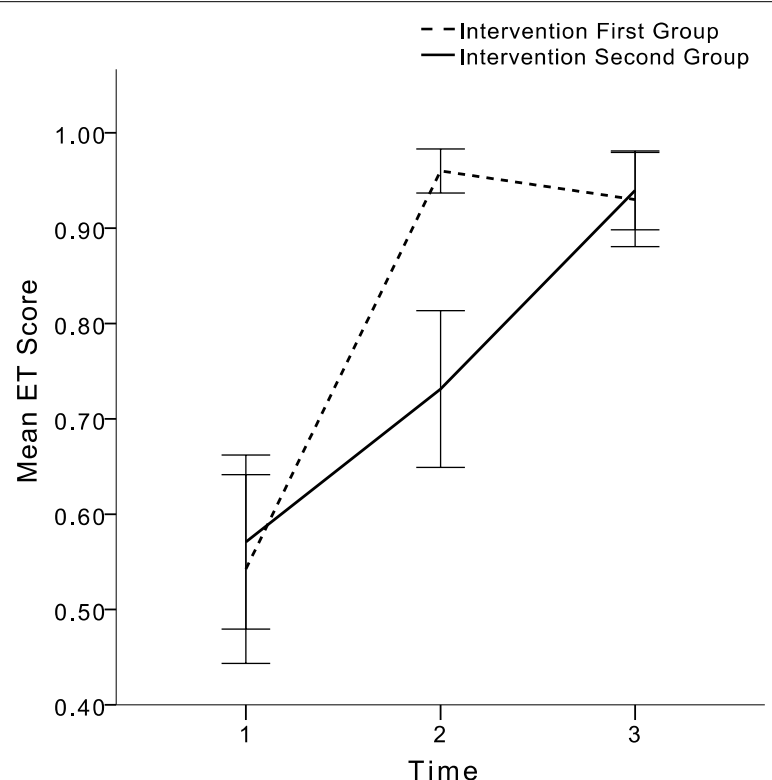

Fig. 3 Mean equivalence test scores as a function of time and intervention group. Means are reported in percent. Error bars represent $\pm 1 \mathrm{SE}$

and time was the within-groups factor. The ANOVA revealed a main effect of time, $F(2,84)=25.64, p<.001$, partial $\eta^{2}=.38$. Because this effect is averaged across groups, who received the intervention at different times, no further post hoc analyses were conducted.

A significant time $\times$ intervention group interaction was also found, $F(2,84)=3.37$, $p=.039$, partial $\eta^{2}=.07$. Tests of simple effects with Bonferroni corrections were conducted to track performance of each group from before to after the intervention. Tests of simple effects also allow for a comparison of the groups at each time point, thus providing evidence of the potential impacts of the intervention. For example, the Intervention Second group would serve as a comparison to the Intervention First group at Time 2, and the Intervention Group First would be the comparison for the Intervention Second group at Time 3 . Therefore, testing both sets of simple effects allowed us to simultaneously provide support for the potential effects of the intervention and reduce the viability of alternate explanations, such as maturation. 


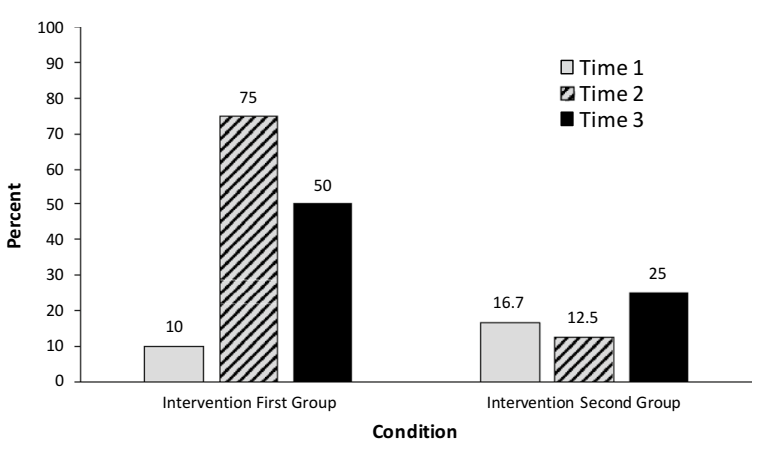

Fig. 4 Percent of students in each intervention group using relational thinking on at least two items of the RTT at each time point

For the Intervention First group, the ET scores were higher at Time 2 compared to Time $1, t(19)=5.23, p<.001, d=1.17$, and this improvement was maintained from Time 2 to Time $3(p>99)$. No improvements were observed between Time 1 and Time 2, $t(23)=2.19, p=.10$, for the Intervention Second group, providing some evidence that the improvement in the Intervention First group between Time 1 and Time 2 was not due to maturation. In addition, the Intervention Second group improved from Time 2 to Time $3, t(23)=3.53, p=.003, d=.72$, showing improvement after the intervention.

No difference between the two groups were observed at Time $1, t(42)=.21, p=.83$, but at Time 2, the Intervention First group outperformed the Intervention Second group, $t(42)=2.46, p=.018, d=.76$, showing a distinct advantage for the group that received the $M M$ intervention relative to the group that did not. After they had received the intervention, students in the Intervention Second group caught up to their peers in the Intervention First group; there was no difference in means at Time $3, t(42)=.16, p=.88$.

\section{Relational thinking test}

To illustrate the extent to which the students in each group engaged in relational thinking on the RTT before and after the mental mathematics intervention, the percent of students in each group whose justifications were coded as Category 1 (relational thinking) on at least half of the items on the RTT (i.e., at least two of the four items) at each time point is presented in Fig. 4. In the Intervention First group, 75\% of the students used relational thinking on at least half of the items on the RTT at Time 2, immediately after the delivery of the intervention. In the Intervention Second group, $25 \%$ of the students' justifications were placed in the Relational Thinking Category on at least 2 of the 4 items at Time 3 , the point after which the intervention was delivered to the group. The proportions were significantly different across time points in the Intervention First group, $\chi^{2}(2, N=20)=17.37, p<.001, \phi=.54$, but not in the Intervention Second group, $\chi^{2}(2, N=24)=1.23, p=.27$. This said, across both groups, $48 \%$ of students used relational thinking on at least half of the RTT items immediately after their respective interventions were delivered.

To verify the hypothesis that students' relational thinking would improve after the MM intervention, we ran a 3-Time (Time 1, Time 2, Time 3) $\times 2$-Intervention Group (Intervention First, Intervention Second) $\times 4$-Operation (addition, subtraction, multiplication, 
and division) mixed ANOVA, with class as the between-groups factor and the other two factors as repeated measures. Mean RTT scores were used as the dependent measure. No effect of operation was found except for a significant time $\times$ operation interaction, $F(6,252)=2.79, p=.012, \eta^{2}=.06$, but this effect is not meaningful given that the means are averaged across groups, who received the intervention at different time points.

Using the RTT means, collapsed across items, as the dependent measure, we ran a 3-Time (Time 1, Time 2, Time 3) $\times 2$-Intervention Group (Intervention First, Intervention Second) mixed ANOVA, with Intervention group as the between-groups factor, and time as the within-groups factor. Significant main effects of Time, $F(2,84)=14.23$, $p<.001$, partial $\eta^{2}=.25$, and Intervention Group, $F(1,42)=9.39, p=.004$, partial $\eta^{2}=.18$, were found. No further post hoc analyses were conducted for the main effect of time because the means are collapsed across groups, who received the intervention at different time points. The main effect of intervention group is also uninterpretable because the means are averaged across time.

A significant time $\times$ class interaction was also found, $F(2,84)=6.75, p=.002$, partial $\eta^{2}=.14$. The means for this interaction are graphed in Fig. 5. Two sets of simple effects tests were conducted to explain the interaction. Similar to the simple effects for the ET, testing both sets of simple effects allowed us to simultaneously provide support for the potential effects of the intervention and reduce the viability of alternate explanations, such as maturation.

Tests of simple effects with Bonferroni corrections indicated that for the Intervention First group, the RTT scores were higher at Time 2 compared to Time $1, t(19)=5.02$, $p<.001, d=1.12$, which shows improved performance after the intervention was delivered. Their performance was not maintained from Time 2 to Time 3 however, as indicated by significantly lower scores at Time $3, t(19)=-2.87, p=.025, d=.62$, but their RTT scores at Time 3 were nevertheless still higher than those at Time 1, before the intervention was delivered, $t(19)=3.18, p=.008, d=.71$. Therefore, the performance of the students in the Intervention First group on the RTT did not go back to baseline (Time 1), despite the dip from Time 2 to Time 3.

For the Intervention Second group, no improvements were observed between Time 1 and Time $2, t(23)=.82, p>.99$, but the students' scores were significantly higher after having received the intervention. That is, students' RTT scores were higher at Time 3 compared to Time $2, t(23)=2.70, p=.03, d=.55$. Similar to the Intervention First group, performance on the RTT in the Intervention Second group was higher at Time 3 than 8 weeks previously at Time $1, t(23)=2.50, p=.048, d=.51$.

No differences between the groups were revealed at Time $1, t(42)=.89, p=.38$, but at Time 2, the Intervention First group outperformed the Intervention Second group, $t(42)=4.82, p<.001, d=1.49$ ), again providing additional evidence that the improvement after the invention was not due to maturation. Both groups had comparable RTT mean scores at Time 3, $t(42)=1.69, p=.10$.

\section{Discussion}

The present study investigated seventh graders' relational thinking after having received a mental mathematics intervention in addition to the regular curriculum. Using two intact seventh-grade classes, students were assessed at three time points on their ability 


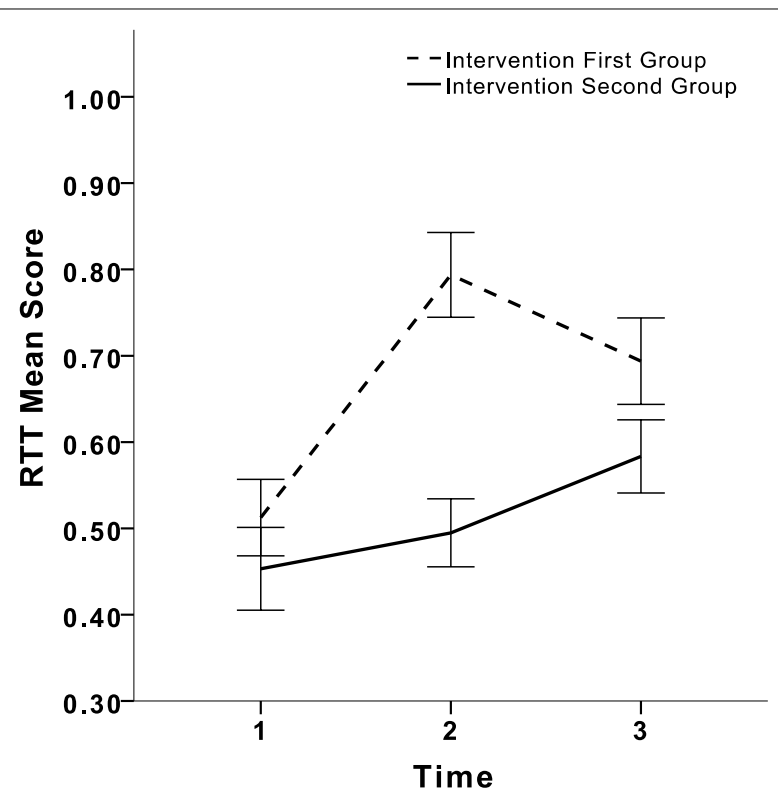

Fig. 5 Mean relational thinking test scores as a function of time and intervention group. Means are reported in percent. Error bars represent $\pm 1 \mathrm{SE}$

to solve equivalence problems and on their reasoning about true-false number sentences. A first class (the Intervention First group) received a mental mathematics intervention, and following its conclusion, the same mental mathematics intervention was delivered to a second class (the Intervention Second group). In line with our predictions, our results revealed that, immediately following the intervention in each class, the students' understanding of the equal sign and their relational thinking improved. Moreover, for the Intervention First group, the level of performance on equivalence problems persisted for 4 weeks after the intervention ended, but the students' relational thinking was not maintained. In light of these results, we speculate a possible causal link between mental mathematics and relational thinking. Future studies with random assignment to groups are nevertheless required to investigate the causal connection more directly.

The improved performance on equivalence problems in both groups has important implications, particularly with respect to relational thinking, as researchers have previously argued that students who struggle to understand the equal sign appear to engage in meaningless computations instead of reflecting on the relationships that exist between numbers $[17,20]$. As such, without viewing the equal sign symbol as a relational one, further relational thinking can be impeded. We speculate that the students in our study improved their understanding of the equal sign because they were encouraged to create equivalent and more manageable expressions when attempting to solve mental mathematics problems. For example, without the explicit use of the equal sign, students could transform $49+51$ into the equivalent expression $50+50$, thereby making the expression more manageable to solve mentally. Despite the fact that the equal sign was not demonstrated while such mathematical transformations were shared and discussed during the intervention, the equal sign is implicit in this transformation, as the two expressions are equivalent and each can be substituted for the other [18]. 
In addition, students' scores on the relational thinking assessment improved immediately following the mental mathematics intervention. We speculate that this improvement can be explained by the overlap in cognitive processes that are implicated in both mental mathematics and relational thinking [13, 14, 45-48]. As indicated previously, at the heart of relational thinking are transformations and substitutions of equivalent numbers and expressions, which are contingent not only on a relational conception of the equal sign, but also on a solid understanding of number properties, such as commutativity and distributivity. We speculate that the students' fluency with the number properties that justified their transformations increased during the mental mathematics intervention and was detected on our measure of relational thinking afterward. Additional data, such as verbal protocols, would elucidate the actual processes that would more clearly explain the cognitive processes that are responsible for the development of relational thinking in the context of mental computation.

Furthermore, during the course of the mental mathematics intervention, the students engaged in whole-class discussions, guided by the teacher, in which they shared the strategies they used to mentally solve a given problem. By explaining their strategies and being exposed to different strategies used by their peers, applications of the fundamental properties of arithmetic were made visible. Moreover, the wide variety of strategies that were presented and discussed during the mental mathematics intervention encouraged students to become more flexible in selecting their own strategies for a given mathematical expression.

The results of the present study can be highlighted by situating them in the current literature. Previous research has shown that at baseline (i.e., without instruction), students at the upper elementary grades hold operational views of the equal sign. Falkner et al. [11], for example, found that as few as $7 \%$ of the fifth graders and no sixth graders were able to find a number that would make $8+4=\square+5$ true. More recently, Li et al. [23] asked Chinese and American sixth graders to fill in the box in noncanonical opennumber sentences (e.g., $6+9=\square+4$ ). Although the Chinese students performed very well on this task, only $28 \%$ of the American students were successful.

Asking students directly what the equal sign means yields similar operational patterns. Alibali et al. [1] gave students the expression $3+4=7$ and asked them to name the symbol between the 4 and 7 and to explain what the symbol meant. Only $20 \%$ of the sixth graders and less than $40 \%$ of the seventh graders gave a relational definition. On the same task, Asquith et al. [3] found that only $29 \%$ of the sixth graders, $37 \%$ of the seventh graders, and $44 \%$ of the eighth graders provided a relational definition when explaining the meaning of the equal sign. In contrast to these studies, we found near ceiling performance on equivalence problem solving after the mental mathematics intervention, suggesting considerably enhanced understanding of the equal sign after the intervention was delivered.

Regarding relational thinking more broadly, previous research also shows that without instruction, middle-school students struggle to reason structurally about quantitative relationships. For example, Stephens and Ribeiro [44] found that very few seventh and eighth graders could consistently reason relationally about noncanonical equations, with the worst performance on relationships involving multiplication and division (e.g., Can you put numbers in these boxes to make the sentence correct? What is the relationship 
between the numbers in the boxes? $5 \times \square=10 \times \square$ ). In a similar study, Stephens [43] asked Japanese, Thai, and Australian middle-school participants to solve open-number sentences with multidigit numbers involving addition (e.g., $23+15=26+\square$ ) and subtraction (e.g., $104-45=\square-46$ ). Most of the students in both the sixth and the seventh grades used computation alone to solve the problems rather than relational thinking. In our study, on the contrary, almost $50 \%$ of the students used relational strategies rather than computation on at least two of the problems on the relational thinking test following the mental mathematics intervention, providing further support for the role of mental computation in enhancing students' relational thinking.

\section{Limitations and future research}

Although our study provides support for the relationship between mental mathematics and relational thinking, there are limitations to the current study. For example, the students who participated in the study were from two intact classes, as random assignment was not feasible. Therefore, it is possible that the results obtained in this study are a result of factors other than the mental mathematics intervention. It is possible, for example, that the two classes may have had pre-existing differences that were not accounted for, such as private mathematics tutoring or previous exposure to algebra, which may have confounded the results. To mitigate this weakness, we designed the study so that each of the two classes had their interventions delivered using a staggered treatment design (i.e., the first class received the intervention while the second class did not, followed by the delivery of the intervention to the second class). This staggered design was intended to moderate the effects of the lack of random assignment by providing some evidence that any observed improvements were not a result of maturation.

Moreover, the first author was the students' regular mathematics teacher, and she was responsible for the delivery of all of the mental mathematics sessions in both classes. Despite her best efforts to deliver comparable instruction in both classes regardless of content (i.e., the mental mathematics intervention or the regular mathematics curriculum), it is possible that experimenter bias may have occurred. For future studies, an observer who is blind to the study's hypotheses could verify, based on predetermined criteria, that the instructional practices of the teacher were equivalent in all groups. Alternatively, counterbalancing instructors who are blind to the research hypotheses across groups that are randomly assigned to mental mathematics and control conditions would be a more stringent test of causality, but would greatly reduce the ecological validity of such a study.

Further to this, the study took place in a public suburban high school with a student population from middle- to high-income families. As such, it is possible that these results are not easily generalizable to other high schools or other populations, as it is conceivable that students from different or less affluent populations may respond differently to the intervention. In the absence of a true experiment with greater external validity, it would be useful in future studies to assess students' mental mathematics abilities before and after the intervention, not only as an index of treatment integrity, but also to verify that the improvements in mental mathematics ability corresponded in time to the improvements on the equivalence and relational thinking assessments. In accordance with our hypothesis, we would predict that patterns of performance on a mental 
mathematics assessment would mirror performance on outcome measures of equivalence and relational thinking.

Finally, further research on ways to assess relational thinking is necessary. In the present study, we did not find sufficient evidence for test-retest reliability for the relational thinking measure we created and used. This suggests that the measure should be modified and tested for validity and reliability. Refinements could be based on existing assessments of relational thinking. For example, the measure used by Stephens and Ribeiro [44] involved number sentences such as $5+\square=6+\square$ to assess the seventh and the eighth graders' relational thinking. Students were required to fill in both blank lines, as well as to explain the reasons for the numbers they selected. In doing so, students were able to justify their relational thinking by means of numbers that made sense to them, as well as describe the direction of the compensation required to create a true number sentence. By means of a problem for which students have some freedom in generating acceptable answers, Stephens and Ribeiro were able to determine if the students understood the relationship between the expressions on either side of the equal sign, which is at the core of relational thinking. Even without justifications, students' responses would provide valuable insight into how they view relationships between numbers.

\section{Educational implications}

Educators can infer from this study that mental mathematics may serve as an effective way to enhance relational thinking at the middle-school level (e.g., the seventh and the eighth grades). As our results suggest, students' abilities in relational thinking improved following a mental mathematics intervention of just 20 min per day over a 15-day period. Therefore, to the extent that the results allow for pedagogical prescriptions, teachers who aim to improve students' understanding of the equal sign and relational thinking should aim to incorporate mental mathematics instruction into their daily mathematics routine.

It may be the case, however, that students need more practice in mental computation to maintain any resulting gains in relational thinking. Unlike students' understanding of the equal sign symbol, which persisted 4 weeks after the intervention concluded in the Intervention First group, students' relational thinking was not maintained over time. This result may be due to the relatively weak psychometric properties of the relational thinking measure used, but could also be attributed to the complex nature of relational thinking relative to what is involved in solving mathematical equivalence problems. Relational thinking requires that students examine a given numerical expression as a unit, and then evaluate the mathematical structure and corresponding components to arrive at a solution [32]. Relational thinking relies on such strategies as transforming, substituting, and applying knowledge of number properties flexibly in a variety of contexts $[9,10,44]$. As such, teachers may wish to increase the length of a mental mathematics intervention, or possibly integrate it with the regular curriculum [40], for observing the long-term gains in relational thinking.

To facilitate student improvement in relational thinking, the results of our study also imply that teachers should have a solid foundation of number properties themselves, and be able to use the equal sign meaningfully to reflect on numerical relationships [17]. From our perspective, educators also need to understand the similarities between 
mental mathematics and relational thinking, as described in the present paper, so that the way in which they encourage mental mathematics in their classrooms can encourage relational thinking. We argue that once this foundation is established, educators should use similar strategies as those used in the present study to improve mental mathematics abilities and to foster relational thinking in their students. During the intervention delivered in this study, the types of classroom discussions, and the ways in which the teacher guided these discussions, appeared critical in creating the links between the mental mathematics strategies used and the processes required for relational thinking. The teacher was instrumental in underscoring the commonalities in the variety of strategies that were presented, and in the implicit use and demonstration of fundamental number properties [15]. A teacher who encourages the creation of equivalent expressions as she directs whole-class discussions toward transforming, rearranging, and substituting various numbers within a problem, is more likely to enhance her students' understanding of equivalence and their ability to reason structurally about mathematical equations.

\section{Abbreviations}

ANOVA: analysis of variance; $d$ : Cohen's measure of sample effect size for comparing two sample means; ET: equivalence test; $M$ : mean; N: number of students; $P$ : probability; PK: prior knowledge; RTT: relational thinking test; SD: standard deviation; $t$ : student's $t$ distribution; a statistical test based on the student $t$ distribution; the sample value of the $t$ test statistic; T1:Time 1;T2:Time 2;T3:Time $3 ; \eta^{2}$ : measure of strength of relationship (eta squared).

\section{Authors' contributions}

Conceptualization and design of the study: ANK, HPO; Data collection: ANK; Data analysis: ANK, HPO; Writing of the paper: ANK, HPO. Both authors read and approved the final manuscript.

\section{Acknowledgements}

The authors wish to acknowledge Emmanuelle Adrien for her assistance in coding the data. A portion of these data were presented at the 2015 meeting of the Jean Piaget Society in Toronto, Canada.

Competing interests

The authors declare that they have no competing interests.

Availability of data and materials

Data supporting this manuscript can be found at Concordia University, Department of Education.

Consent for publication

Parental and student consent for publication were obtained for all data in this manuscript.

Ethics approval and consent to participate

This study was approved by the Concordia University Human Research Ethics Committee. Parental consent and student assent were obtained for all participants.

Funding

No funding for this study.

\section{Publisher's Note}

Springer Nature remains neutral with regard to jurisdictional claims in published maps and institutional affiliations.

Received: 7 February 2017 Accepted: 14 October 2017

Published online: 23 April 2018

\section{References}

1. Alibali, M.W., Knuth, E.J., Hattikudur, S., McNeil, N.M., Stephens, A.C.: A longitudinal examination of middle school students' understanding of the equal sign and equivalent equations. Math. Think. Learn. 9(3), 221-247 (2007)

2. Ambrose, R., Baek, J., Carpenter, T.P.: Children's invention of multidigit multiplication and division algorithms. In: Baroody, A.J., Dowker, A. (eds.) The development of arithmetic concepts and skills: constructive adaptive expertise, pp. 305-336. Erlbaum, Mahwah (2003)

3. Asquith, P., Stephens, A.C., Knuth, E.J., Alibali, M.W.: Middle school mathematics teachers' knowledge of students' understanding of core algebraic concepts: equal sign and variable. Math. Think. Learn. 9(3), 249-272 (2007) 
4. Baroody, A.J., Ginsburg, H.P.: The effects of instruction on children's understanding of the "equals" sign. Elem. School J. 84(2), 199-212 (1983)

5. Behr, M., Erlwanger, S., Nichols, E.: How children view the equals sign. Math. Teach. 92, 13-15 (1980)

6. Britt, M., Irwin, K.: Algebraic thinking with and without algebraic representation: A pathway for learning. In: Cai, J., Knuth, E. (eds.) Early algebraization: a global dialogue from multiple perspectives, pp. 137-160. Springer, Dordrecht (2011)

7. Capraro, M.M., Ding, M., Matteson, S., Capraro, R.M., Li, X.: Representational implications for understanding equivalence. School Sci. Math. 107(3), 86-88 (2007)

8. Carpenter, T.P., Franke, M.L., Levi, L.: Thinking mathematically: integrating arithmetic and algebra in elementary school. Heinemann, Portsmouth (2003)

9. Carpenter, T.P., Levi, L., Franke, M.L., Zeringue, J.K.: Algebra in elementary school: developing relational thinking. Zentralblatt für Didaktik der Mathematik 37(1), 53-59 (2005)

10. Empson, S.B., Levi, L., Carpenter, T.P.: The algebraic nature of fractions: developing relational thinking in elementary school. In: Cai, J., Knuth, E. (eds.) Early algebraization: a global dialogue from multiple perspectives, pp. 409-426. Springer, New York (2011)

11. Falkner, K.P., Levi, L., Carpenter, T.P.: Children's understanding of equality: a foundation for algebra. Teach. Child. Math. 6(4), $232(1999)$

12. Freiman, V., Lee, L.: Tracking primary students' understanding of the equal sign. In: Johnsen M., Berit A. (Eds.) Proceedings of the 28th international group for the psychology of mathematics education, vol. 2, pp 415-422. Bergen University College, Bergen (2004)

13. Heirdsfield, A.M.:Teaching mental computation strategies in early mathematics. Young Child. 66(2), 96-102 (2011)

14. Heirdsfield, A.M., Cooper, T.J.: Flexibility and inflexibility in accurate mental addition and subtraction: two case studies. J. Math. Behav. 21(1), 57-74 (2002)

15. Hiebert, J., Grouws, D.A.:The effects of classroom mathematics teaching on students'learning. In: Lester, F.K. (ed.) Second handbook of research on mathematics teaching and learning, pp. 371-404. NCTM, Reston (2007)

16. Irwin, K.C., Britt, M.S.: The algebraic nature of students'numerical manipulation in the New Zealand Numeracy Project. Educ. Stud. Math. 58(2), 169-188 (2005)

17. Jacobs, V.R., Franke, M.L., Carpenter, T.P., Levi, L., Battey, D.: Professional development focused on children's algebraic reasoning in elementary school. J. Res. Math. Educ. 38(3), 258-288 (2007)

18. Jones, I., Inglis, M., Gilmore, C., Evans, R.: Teaching the substitutive conception of the equals sign. Res. Math. Educ. 15(1), 34-49 (2012)

19. Kieran, C.: Concepts associated with the equality symbol. Educ. Stud. Math. 12, 317-326 (1981)

20. Kieran, C.: The learning and teaching of school algebra. In: Grouws, D.A. (ed.) Handbook of research on mathematics teaching and learning, pp. 390-419. Macmillan, New York (1992)

21. Knuth, E.J., Alibali, M.W., McNeil, N.M., Weinberg, A., Stephens, A.C.: Middle school students' understanding of core algebraic concepts: equivalence \& variable 1. ZDM 37(1), 68-76 (2005)

22. Knuth, E.J., Stephens, A.C., McNeil, N.M., Alibali, M.W.: Does understanding the equal sign matter? Evidence from solving equations. J. Res. Math. Educ. 37(4), 297-312 (2006)

23. Li, X., Ding, M., Capraro, M.M., Capraro, R.M.: Sources of differences in children's understandings of mathematical equality: comparative analysis of teacher guides and student texts in China and the United States. Cognit. Instr. 26(2), 195-217 (2008)

24. Maclellan, E.: Mental calculation: its place in the development of numeracy. Westminster Stud. Educ. 24(2), 145-154 (2001)

25. Markovits, Z., Sowder, J.: Developing number sense: an intervention study in grade 7. J. Res. Math. Educ. 25(1), 4-29 (1994)

26. McNeil, N.M.: A change-resistance account of children's difficulties understanding mathematical equivalence. Child Dev. Perspect. 8(1), 42-47 (2014)

27. McNeil, N.M., Alibali, M.W.: Knowledge change as a function of mathematics experience: all contexts are not created equal. J. Cognit. Dev. 6, 285-306 (2005)

28. McNeil, N.M., Grandau, L., Knuth, E.J., Alibali, M.W., Stephens, A.C., Hattikudur, S., Krill, D.E.: Middle-school students' understanding of the equal sign: the books they read can't help. Cognit. Instr. 24(3), 367-385 (2006)

29. Ministère de l'éducation, enseignement supérieur, et recherche. Compilation spéciale des données du recensement canadien. http://www.education.gouv.qc.ca/fileadmin/site_web/documents/PSG/statistiques_info_decisionnelle/ Indices_defavorisation_ecoles_2014_2015.pdf (2014). Accessed 5 May 2017

30. Ministère de l'éducation, enseignement supérieur, et recherche. Quebec Education Program. Retrieved from http:// www1.education.gouv.qc.ca/sections/programmeFormation/secondaire1/index_en.asp (2016). Accessed 5 May 2017

31. Molina, M., Ambrose, R.: What is that equal sign doing in the middle? Fostering relational thinking while negotiating the meaning of the equal sign. Teach. Child. Math. 13(2), 111-117 (2006)

32. Molina, M., Castro, E., Mason, J.: Elementary students'approaches to solving true/false number sentences. Revista de Investigacion en Didactica de la Matematica PNA 2(2), 75-86 (2008)

33. National Council of Teachers of Mathematics: Principles and standards for school mathematics. Author, Reston (2000)

34. Osana, H.P., Proulx, J., Adrien, E., Nadeau, D.: Developing relational thinking in preservice teachers. In: Martinez, M., Castro Superfine, A. (eds.) Proceedings of the 35th annual meeting of the North American chapter of the international group for the psychology of mathematics education, pp. 845-847. University of Illinois at Chicago, Chicago (2013)

35. Paholek, E.: Canadian mathematics 7. Rational Publications, Edmonton (1993)

36. Perry, M.: Learning and transfer: instructional conditions and conceptual change. Cognit. Dev. 6(4), 449-468 (1991)

37. Proulx, J.: Mental mathematics, emergence of strategies, and the enactivist theory of cognition. Educ. Stud. Math. 84(3), 309-328 (2013) 
38. Rittle-Johnson, B., Matthews, P.G., Taylor, R.S., McEldon, K.L.: Assessing knowledge of mathematical equivalence: a construct-modeling approach. J. Educ. Psychol. 103, 85-104 (2011)

39. Sherman, J., Bisanz, J.: Equivalence in symbolic and nonsymbolic contexts: benefits of solving problems with manipulatives. J. Educ. Psychol. 101(1), 88 (2009)

40. Sowder, J.: Estimation and number sense. In: Grouws, D.A. (ed.) Handbook of research on mathematics teaching and learning, pp. 371-389. Macmillan, New York (1992)

41. Spielhagen, F.R.: Closing the achievement gap in math: the long-term effects of eighth—grade algebra. J. Adv. Acad. 18(1), 34-59 (2006)

42. Steinberg, R.M., Sleeman, D.H., Ktorza, D.: Algebra students'knowledge of equivalence of equations. J. Res. Math Educ. 22, 112-121 (1991)

43. Stephens, M. Students' emerging algebraic thinking in the middle school years. In: Watson J., Beswick K. (eds.) Mathematics: essential research, essential practice. Proceedings of the 30th annual conference of the mathematics education research group of Australasia, pp. 678-687. MERGA, Adelaide (2007)

44. Stephens, M., Ribeiro, A.: Working towards algebra: the importance of relational thinking. Revista Latinoamericano de Investigacion en Matematica Educativa 15(3), 373-402 (2012)

45. Stephens, M., Wang, X.: Investigating some junctures in relational thinking: a study of year 6 and year 7 students from Australia and China. J. Math. Educ. 1(1), 28-39 (2008)

46. Threlfall, J.: Flexible mental calculation. Educ. Stud. Math. 50, 29-47 (2002)

47. Thompson, l.: Getting your head around mental calculation. In: Thompson, I. (ed.) Issues in teaching numeracy in primary schools, pp. 145-156. Open University Press, New York (2010)

48. Verschaffel, L., Luwel, K., Torbeyns, J., Van Dooren, W.: Conceptualizing, investigating, and enhancing adaptive expertise in elementary mathematics education. Eur. J. Psychol. Educ. 24(3), 335-359 (2009)

\section{Submit your manuscript to a SpringerOpen ${ }^{\circ}$ journal and benefit from:}

- Convenient online submission

- Rigorous peer review

- Open access: articles freely available online

- High visibility within the field

- Retaining the copyright to your article

Submit your next manuscript at $>$ springeropen.com 